\title{
Usage of statistical cues for word boundary in reading Chinese sentences
}

\author{
Miao-Hsuan Yen • Ralph Radach • \\ Ovid J.-L. Tzeng $\cdot$ Jie-Li Tsai
}

(C) Springer Science+Business Media B.V. 2011

\begin{abstract}
The present study examined the use of statistical cues for word boundaries during Chinese reading. Participants were instructed to read sentences for comprehension with their eye movements being recorded. A two-character target word was embedded in each sentence. The contrast between the probabilities of the ending character $(\mathrm{C} 2)$ of the target word (C12) being used as word beginning and ending in all words containing it was manipulated. In addition, by using the boundary paradigm, parafoveal overlapping ambiguity in the string C123 was manipulated with three types of preview of the character $\mathrm{C} 3$, which was a singlecharacter word in the identical condition. During preview, the combination of C23' was a legal word in the ambiguous condition and was not a word in the control condition. Significant probability and preview effects were observed. In the lowprobability condition, inconsistency in the frequent within-word position (word beginning) and the present position (word ending) lengthened gaze durations and increased refixation rate on the target word. Although benefits from the identical previews were apparent, effects of overlapping ambiguity were negligible. The results suggest that the probability of within-word positions had an influence during
\end{abstract}

M.-H. Yen · O. J.-L. Tzeng

Laboratories for Cognitive Neuroscience, Institute of Neuroscience, National Yang-Ming University, Taipei, Taiwan

M.-H. Yen · J.-L. Tsai ( $₫)$

Department of Psychology and Research Center for Mind, Brain, and Learning,

National Chengchi University, Taipei, Taiwan

e-mail: jltsai@nccu.edu.tw

R. Radach

General and Biological Psychology, University of Wuppertal, Wuppertal, Germany

O. J.-L. Tzeng

The Institute of Linguistics, Academia Sinica, Taipei, Taiwan 
character-to-word assignment, which was mainly verified during foveal processing. Thus, the overlapping ambiguity between parafoveal words did not interfere with reading. Further investigation is necessary to examine whether current computational models of eye movement control should incorporate statistical cues for word boundaries together with other linguistic factors in their word processing system to account for Chinese reading.

Keywords Chinese reading - Eye movements · Word processing ·

Word segmentation cues

\section{Introduction}

Reading involves not only linguistic processing but also visual processing and oculomotor control. Because visual acuity decreases from the fovea to periphery, readers actively move their eyes to sample information for recognition and comprehension (Findlay \& Gilchrist, 2003). The pattern of eye movements during reading is a series of alternate fixations (during which the eyes remain relatively stable for about $250 \mathrm{~ms}$ ) and saccades (during which the eyes move rapidly about 20-50 ms). Linguistic factors, such as word frequency (Inhoff, 1984; Rayner \& Duffy, 1986; Schilling, Rayner, \& Chumbley, 1998) and contextual predictability (e.g., Rayner \& Well, 1996), were found to influence fixation durations, suggesting an immediate processing of the fixated word. In addition, masking the words to the right of the fixated word reduces reading rate to $60 \%$ of that when the whole line of text is visible (Rayner, Well, Pollatsek, \& Bertera, 1982), indicating that information obtained from the parafovea is necessary and helpful for reading. The reduction in fixation durations when the parafoveal word is available compared to when it is masked is generally referred to as parafoveal preview benefit (Rayner, 1998). Thus, not only the fixated word but also the parafoveal word(s) can be processed during each fixation. The region within which visual information can be obtained during each fixation, the perceptual span, is about 4 letters to the left and 14-15 letters to the right of the fixation point in reading alphabetic scripts (Rayner, Well, \& Pollatsek, 1980; Rayner, et al., 1982). General word shape and inter-word spaces can be obtained from the entire span (McConkie \& Rayner, 1975; Rayner, 1975), while letter identity can be extracted from 6-8 letters to the right of the fixation point (Underwood \& McConkie, 1985). Because inter-word spaces explicitly delineate words in the perceptual span, readers of most alphabetic scripts can select words as saccade targets based on low level (low spatial frequency) information (McConkie, Kerr, Reddix \& Zola, 1988). This word-based targeting assumption is supported by the observation of a preferred viewing location (PVL; Rayner, 1979) to the left of the word center.

During the last decade, computational models were developed to account for the underlying mechanism of eye movement control in reading. Model assumptions differ along two main dimensions: (1) whether processing of words in the perceptual span is strictly sequential or partially parallel (i.e., overlaps in time) and (2) whether linguistic processing triggers saccades or modulates autonomous saccade generation 
(Engbert, Nuthmann, Richter, \& Kliegl, 2005; Pollatsek, Reichle, \& Rayner, 2006; Reilly \& Radach, 2006; see Radach, Reilly, \& Inhoff, 2007, for a comprehensive discussion). Despite the different viewpoints, these models generally assume wordbased processing and hence incorporate similar word-based saccade targeting mechanisms.

However, there are scripts, such as Chinese, Japanese, and Thai, in which words are not delimitated by inter-word spaces. How word boundaries are delineated and how word-based processing is executed in these scripts are intriguing questions. In fact, Rayner, Li, and Pollatsek (2007) have recently extended the E-Z Reader model (one of the dominant models) to Chinese reading, maintaining the assumptions of word-based attention shift and saccade targeting. Nevertheless, further investigation about the mechanism of word recognition and the interaction between character and word processing in Chinese reading is necessary. Addressing one critical component of the problem, the present study aimed to investigate the usage of word boundary cues during reading in Chinese.

\section{Eye movements in reading Chinese}

Chinese is a morphosyllabic script. A square-like character corresponds to a syllable and most characters have their own meaning(s). Regardless of visual complexity, each character occupies the same size of space. According to the Academia Sinica balanced corpus (1998), there are 9.5, 65.6, 12.4, and $11.6 \%$ of 54,393 unique words that consist of one, two, three and four characters, respectively. Concerning their frequencies of occurrence, one- and two-character words occur 53.8 and $42.2 \%$ of time among 10 million word counts. Regardless of word length, words are concatenated and written character by character. Thus, Chinese sentences are characterized by visually distinctive characters without physical cues for word boundaries. Nevertheless, both word and character properties were found to influence eye movement during foveal and parafoveal processing. First, high frequency and highly predictable words are fixated more briefly and more likely to be skipped than low frequency and less predictable words (Rayner, Li, Juhasz, \& Yan, 2005; Yan, Tian, Bai, \& Rayner, 2006; Yang \& McConkie, 1999). Tsai, Lee, Lin, Tzeng, and Hung (2006) also found an effect of orthographic neighborhood size (i.e., number of words that differ in one character), in which words with more neighbors are fixated more briefly and more frequently skipped. Similarly, effects of character frequency and complexity (in terms of number of strokes) were observed (Yan, et al., 2006; Yang \& McConkie, 1999). These findings suggest that both character- and word-level information of the fixated stimulus can be extracted during fixation and influence eye movements. Second, a parafoveal lexicality effect has been observed (Yang, Wang, Xu, \& Rayner, 2009; Yen, Tsai, Tzeng, \& Hung, 2008). Masking the parafoveal word (the target) with a pseudoword consisting of real characters lengthened the subsequent target viewing durations. These findings imply that parafoveal information beyond the character level (i.e., orthographical, phonological, and semantic properties of the target characters; Liu, Inhoff, Ye, \& Wu, 2002; Tsai, Lee, Tzeng, Hung, \& Yen, 2004; Yan, Richter, Shu, \& Kliegl, 2009) can be obtained from the parafovea. Attempts to 
demonstrate a preferred viewing location in Chinese had mixed results, with initial corpus based studies reporting no clear PVL (Tsai \& McConkie, 2003; Yang \& McConkie, 1999). However, in more recent analyses, Yan, Kliegl, Richter, Nuthmann, and Shu (2010) observed a PVL on words receiving single fixations, suggesting that word length information can be obtained from the parafovea and used to guide eye movements. In sum, similar to other languages, fixation durations on words are influenced by the properties of the foveal words and their constituent characters. In addition, word information can be extracted during parafoveal preview to affect subsequent viewing durations and landing positions.

\section{Word segmentation cues}

How do Chinese readers segment words in the continuous text without physical cues for word boundaries? Are there linguistic cues available for Chinese readers? Yen, Radach, Tzeng, Hung, and Tsai (2009) analyzed the Academia Sinica balanced corpus (1998) and found that only $17.8 \%$ of 5,915 unique characters can unambiguously signal word boundaries. That is, these characters appear in only one within-word position, namely, single-character words, the beginning characters, or ending characters of multi-character words. The total frequency of their occurrence, 1146.9 per million, is fairly low. In contrast, about $49.1 \%$ of characters can be used in all within-word positions. These characters are fairly frequent $(M=330.7$ per million, $\mathrm{SD}=1087.6$, range $.5-38622.2$ per million $)$ and the total frequency of their occurrence is 960976.9 per million. The fact that most characters are associated with at least two within-word positions can occasionally result in overlapping ambiguity. For example, according to the corpus, about 3.6\% of time, both $\mathrm{C} 12$ and $\mathrm{C} 23$ are legal words in a three-character string C123. Whether $\mathrm{C} 2$ should be combined with $\mathrm{C} 1$ or C3 depends on context. Inhoff and Wu (2005) have observed that all possible combinations (C12 and C23) can be activated and impede the process of the character string. In their study, ambiguous four-character strings (e.g., 專科學生, 'college student') that contained three overlapping two-character words (專科, 'college', 科學, 'science', and 學生, 'student') were fixated longer than unambiguous strings (e.g., 專科老師, 'college teacher') that contained two non-overlapping words (專科, 'college' and 老師, 'teacher').

Although only a minority of characters can unambiguously signal word boundary, the unique profile with respect to how often a character appears in each of the possible within-word positions may be a useful cue for word boundaries. For example, a character which is more frequently used as word ending than as word beginning may suggest that a word boundary to its right is likely. In contrast, it may be difficult to determine the word boundary if a character frequently used as word beginning is actually the end of the present word. The present study aimed to investigate whether the contrast between the probabilities of each character being used as word beginning and word ending (positional probabilities) plays a role in word recognition during Chinese reading.

Recently, effects of linguistic boundary cues have been observed in Thai and Finnish. Reilly, Radach, Luksaneeyanawin, and Aranyanak (2009) found in Thai, an 
alphabetic script without inter-word spaces, that the probabilities of a character being used in the first, middle, and last character positions significantly influenced initial landing position on a word (average word length: 3.5 characters). Landing positions shifted rightwards if the first character is frequently used as the first character and the middle characters are frequently used as middle characters. They shifted leftwards if the last character is frequently used as the last character.

In a more detailed analysis, Bertram, Pollatsek, and Hyönä (2004) investigated specific constituent boundary cues for Finnish two-constituent compound words. First, vowel harmony can signal constituent boundaries. That is, two adjacent vowels with different qualities (front vs. back vowels) have to be separated into two constituents. Gaze durations on compound words with different-quality vowels were shorter than words with same-quality vowels. This effect was persistent even if these two vowels were separated by one consonant. Second, whether or not this consonant can be the end of a constituent also affected target gaze durations. If the consonant cannot be the end of a constituent (i.e., it must be the beginning of the second constituent), the compound words were fixated more briefly than if the consonant can be either the beginning or the end of a constituent. Furthermore, Bertram et al. observed the effect of segmentation cues only (1) when the first constituent was long and (2) on second to fourth fixations on the target. These findings suggest that segmentation cues are used when the constituents are being encoded rather than before they are processed.

In the present study, a two-character word (C12) was embedded in each sentence as the target word. There were two critical manipulations. First, the contrast between the probabilities of the ending character $(\mathrm{C} 2)$ of the target $(\mathrm{C} 12)$ being used as word beginning and ending (hereafter, positional probability) was manipulated. In the low-probability condition, $\mathrm{C} 2$ is more frequently used as word beginning and less likely used as word ending; it may be difficult to process the target word because of the inconsistency between the frequent within-word position (word beginning) and the present position (word ending). Target viewing durations in the low-probability condition were expected to be longer than those in the highprobability condition in which $\mathrm{C} 2$ is more frequently used as word ending.

Second, an overlapping ambiguity between parafoveal words was created by using the boundary paradigm (Rayner, 1975). An invisible boundary was set prior to the target word as the trigger point for display change. In the original sentence, the character (C3) to the right of the target word was a single-character word. Before participants fixated the target, a preview character (C3') was presented at the location of $\mathrm{C} 3$. After participants moved their eyes across the boundary prior to the target word, the original C3 was presented. In the ambiguous condition, the combination of the ending character (e.g., 消) of the target word (e.g., 取消, 'cancel') and the preview character (e.g., 失) was a word (消失, 'disappear'). That is, during preview, the three-character string 取消失 contained two overlapping words 取消 and 消失. In the control condition, the combination of C2 and the preview character (e.g., 必) was not a word. In the identical condition (ID), the original C3 (對) was available before and after the target was fixated. Although preview benefits (comparing identical with non-identical previews) can be expected, it is intriguing to ask whether or not the existence of an overlapping ambiguity 
between parafoveal words is interfering beyond simply blocking the preview of the original C3 (i.e., longer viewing durations in the ambiguous than those in the control condition; c.f. Inhoff \& Wu, 2005).

The present study aimed to investigate (1) the effect of positional probability of $\mathrm{C} 2$ on target processing, (2) whether the overlapping ambiguity between parafoveal words has an effect, and (3) whether this ambiguity effect is modulated by the positional probability of $\mathrm{C} 2$. In general, probability effects were expected. Viewing durations on the target word should be longer if its ending character is more frequently used as word beginning (the low-probability condition) than if it is more frequently used as word ending (the high-probability condition). In addition, overlapping ambiguity between parafoveal words might be interfering, resulting in longer target viewing durations in the ambiguous condition than those in the control condition. Furthermore, if the ending character C2 is more frequently used as word beginning, it may be easier to activate another word that begins with $\mathrm{C} 2$ than if $\mathrm{C} 2$ is more frequently used as word ending. Thus, the effect of overlapping ambiguity should be larger in the low-probability condition than that in the high-probability condition.

\section{Method}

\section{Participants}

Twenty-seven college and graduate students at National Chengchi University and nearby community were paid to participate in the experiment. All of them are native speakers of Chinese with normal or corrected-to-normal vision.

Design and materials

The boundary paradigm was adopted in a 2 by 3 factorial design. The targets were two-character words. The first independent variable was the positional probability of the ending character $(\mathrm{C} 2)$ of the target word (C12). In the low-probability condition, $\mathrm{C} 2$ is frequently used as word beginning and less likely used as word ending in all words including it. In the high-probability condition, C2 is frequently used as word ending and less likely used as word beginning. The second independent variable was preview type. In the sentence, the character (C3) to the right of the target word was a single-character word. Before the target was fixated, a preview character (C3') was presented at the location of $\mathrm{C} 3$. Whether or not the preview character could be combined with the ending character of the target to form an overlapping word (C23) was manipulated. In the identical (ID) condition, C3 was a single-character word and C23 was not a word. The combination C23' was a word in the ambiguous condition but C23" was not a word in the control condition.

The positional probability of each critical character (C2), which is defined as the contrast between the probabilities of $\mathrm{C} 2$ being used as word beginning and ending, was calculated from the Academia Sinica balanced corpus (1998). First, separately for each within-word position (such as word beginning and ending), number of words 
Table 1 The means of positional probabilities (calculated by token and type frequencies) and number of position-specific neighbors (when $\mathrm{C} 2$ was used as word ending and beginning) in each probability condition

\begin{tabular}{|c|c|c|c|c|}
\hline \multirow{2}{*}{$\begin{array}{l}\text { Positional } \\
\text { probability }\end{array}$} & \multicolumn{2}{|l|}{ Probability } & \multicolumn{2}{|c|}{ Number of position-specific neighbors } \\
\hline & $\begin{array}{l}\text { By token } \\
\text { frequency }\end{array}$ & $\begin{array}{l}\text { By type } \\
\text { frequency }\end{array}$ & $\begin{array}{l}\mathrm{C} 2 \text { as word } \\
\text { ending }(\mathrm{C} 12)\end{array}$ & $\begin{array}{l}\mathrm{C} 2 \text { as word } \\
\text { beginning }\left(\mathrm{C} 23^{\prime}\right)\end{array}$ \\
\hline Low & 14.9 & 20.6 & 8.4 & 34.6 \\
\hline High & 85.0 & 80.6 & 41.5 & 8.5 \\
\hline
\end{tabular}

that contain C2 (type frequency) at the corresponding position was computed. Then, the positional probability was calculated by dividing the number of two-character words in which $\mathrm{C} 2$ is used as word ending by the number of two-character words that contain C2. Similarly, the positional probability was also calculated in terms of token frequency, which is the sum of frequencies of two-character words that contain C2 at a specific position. Two different sets of targets were selected for the low- and highprobability conditions, respectively. In the low-probability condition, the probability of $\mathrm{C} 2$ being used as word ending is lower than $30 \%$ in terms of token frequency and lower than $40 \%$ in terms of type frequency (Table 1). Furthermore, numbers of position-specific neighbors were controlled. Specifically, number of words (C12 candidates) in which $\mathrm{C} 2$ is used as word ending was restricted to the range of 2 to 15 $(M=8.4)$. In contrast, number of words ( $\mathrm{C} 23^{\prime}$ candidates) in which $\mathrm{C} 2$ is used as word beginning was higher than $15(M=34.6)$ and was at least twice as large as number of $\mathrm{C} 12$ candidates for each critical character. The contrast between numbers of position-specific neighbors was significant $(p<.001)$. On the other hand, in the high-probability condition, the probability of C2 being used as word ending is higher than $70 \%$ in terms of token frequency and higher than $60 \%$ in terms of type frequency. Following the same but opposite criteria, the contrast between number of position-specific neighbors (C12: 41.5 and $\mathrm{C}^{2} 3^{\prime}$ : 8.5) was also significant $(p<.001)$. The numbers of position-specific neighbors also differed significantly between probability conditions $(p s<.001)$. Furthermore, the probability of predicting C2 from C1 (i.e., forward transitional probability; McDonald \& Shillcock, 2003) did not differ between conditions (7.9 and 5.7\%, respectively for the low- and high-probability conditions, $p>.21)$.

There were 72 items in each probability condition. As is shown in Table 2, frequencies of the target words were not significantly different between the lowprobability $(M=11.5$ per 1 million, $\mathrm{SD}=16.0$, range $2.1-114.7)$ and the highprobability $(M=11.4$ per 1 million, $\mathrm{SD}=11.2$, range 2.1-55.9) conditions, $p>.94$. For each target word, there was an overlapping word $\left(\mathrm{C} 23^{\prime}\right)$ for the ambiguous condition from the same word class (noun or verb) ${ }^{1}$ and of similar word

\footnotetext{
${ }^{1}$ Characters that are more frequently used as word beginning tend to form verbs when combined with other characters regardless of within-word positions. In contrast, characters that are more frequently used as word ending tend to form nouns. In the present study, C12 and C23' were chosen from the same word classes (noun or verb). Thus, a large proportion of target words in the low-probability condition were verbs but a small proportion of target words in the high-probability condition were verbs. The difference between nouns and verbs during reading Chinese sentences has not been documented in the literature to
} 
Table 2 Examples and properties of the target and the preview stimuli

\begin{tabular}{|c|c|c|c|c|}
\hline \multirow[t]{2}{*}{ Positional probability } & \multirow[t]{2}{*}{ Target } & \multicolumn{3}{|l|}{ Preview type } \\
\hline & & Identical & Ambiguous & Control \\
\hline \multicolumn{5}{|l|}{ Example } \\
\hline Low & 取消 & 對 / 消對 & 失 / 消失 & 必 / 消必 \\
\hline High & 綽號 & 較 / 號較 & 碼 / 號碼 & 輝 / 號輝 \\
\hline \multicolumn{5}{|l|}{ Word meaning } \\
\hline Low & 'to cancel' & - & 'to disappear' & - \\
\hline High & 'nickname' & - & 'number' & - \\
\hline \multicolumn{5}{|l|}{ Meaning of $C 3$} \\
\hline Low & & 'concerning' & 'to lose' & 'must' \\
\hline High & & 'compare to' & 'code' & 'splendor' \\
\hline \multicolumn{5}{|l|}{ Mean word frequency } \\
\hline Low & 11.5 & - & 12.2 & - \\
\hline High & 11.4 & - & 12.6 & - \\
\hline \multicolumn{5}{|l|}{ Mean no. of strokes } \\
\hline Low & $\mathrm{C} 1 / \mathrm{C} 2$ & $\mathrm{C} 3$ & C3' & C3, \\
\hline \multirow[t]{2}{*}{ High } & $11.6 / 11.9$ & 7.8 & 11.9 & 11.9 \\
\hline & $11.7 / 12.3$ & 8.6 & 11.7 & 11.7 \\
\hline \multicolumn{5}{|c|}{ Mean character frequency } \\
\hline Low & $522 / 199$ & 13848 & 425 & 438 \\
\hline High & $528 / 344$ & 11551 & 763 & 669 \\
\hline \multicolumn{5}{|c|}{ Mean subjective familiarity (1-7) } \\
\hline Low & $5.3 / 5.3$ & 6.6 & 5.5 & 5.4 \\
\hline High & $5.4 / 5.3$ & 6.5 & 5.5 & 5.4 \\
\hline
\end{tabular}

Word frequency: 1 million words. Character frequency: 1 million characters

\section{Footnote 1 continued}

our knowledge. We restricted the analysis to 23 frequency-matched verbs in both probability conditions and found similar results as those in the main analysis. Critically, the gaze durations in the area C123 and the target $\mathrm{C} 12$ were significantly longer in the low-probability condition than those in the high-probability condition $\left[\mathrm{C} 123: 25 \mathrm{~ms} ; F_{1}(1,26)=4.96, M S_{\mathrm{e}}=5187, p<.05 ; F_{2}(1,44)=1.69, M S_{\mathrm{e}}=14587\right.$, $p>.20 ; \mathrm{C} 12: 42 \mathrm{~ms} ; F_{1}(1,26)=23.43, M S_{\mathrm{e}}=2983, p<.001 ; F_{2}(1,44)=7.48, M S_{\mathrm{e}}=10080$, $p<.01]$. There was no difference in FFD $(F \mathrm{~s}<1.32, p \mathrm{~s}>.26)$ between probability conditions. However, the refixation rate in the low-probability condition was significantly higher than that in the high-probability condition on the target $\mathrm{C} 12\left[F_{1}(1,26)=13.09, M S_{\mathrm{e}}=240, p<.01 ; F_{2}(1,44)=7.95\right.$, $\left.M S_{\mathrm{e}}=468, p<.01\right]$ but the difference was not significant in the area $\mathrm{C} 123(F \mathrm{~s}<1.31, p \mathrm{~s}>.26)$. The results of the analysis restricted to nouns are not reliable since only 8 frequency-matched nouns can be found. In addition, we analyzed gaze durations on all two-character words in the sentences in the present study in a supplementary linear mixed-effects analysis (Baayen, 2008). Both effects of word class and positional probability were observed. Gaze durations on verbs were longer than those on nouns $(t=4.89)$. In addition, the higher the probability of being used as word beginning was, the longer the gaze durations $(t=2.06)$. In addition, the effect of positional probability was larger for verbs than that for nouns $(t=1.89)$. Further studies are necessary for a deep understanding of the effect of positional probability on word recognition. 
frequency. Frequencies of the overlapping words were not significantly different between the low-probability $(M=12.2$ per 1 million, $\mathrm{SD}=14.7$, range $2.1-83.2$ ) and high-probability $(M=12.6$ per 1 million, $\mathrm{SD}=14.9$, range $2.1-82.6$ ) conditions, $p>.89$. The character $\left(\mathrm{C}^{\prime \prime}\right)$ to the right of the target in the control condition was matched to that $\left(\mathrm{C}^{\prime}\right)$ in the ambiguous condition in terms of number of strokes and character frequency. 2,3

Each target word (C12) was embedded in the middle part of a sentence frame and the context preceding the target was neutral. The contextual predictability of the target was assessed by a cloze task. Ten participants who did not participate in the experiment were instructed to fill in a word that can continue the sentence fragment prior to the target. The predictability of the target word was low (2.6 and $2.4 \%$ in the low- and high-probability conditions, respectively) and was not different between conditions $(p>.76)$. In addition, the ambiguous previews $\left(\mathrm{C}_{2} 3^{\prime}\right)$ were chosen to be contextually inconsistent with the sentences so that they would be interfering. The contextual fit of the $\mathrm{C} 12$ and $\mathrm{C} 23^{\prime}$ were assessed by three additional participants. They were presented with the sentence fragment prior to the target together with either $\mathrm{C} 12$ or $\mathrm{C}_{23}{ }^{\prime}$ and were instructed to assess how well the presented word fit into the sentence with a 5-point scale (5: the word fits into the sentence very well; 1 : the word does not fit into the sentence at all). The contextual fit of the target words $(M=4.0)$ was significantly higher than that of the ambiguous preview (C23', $M=2.0), p<.001$. Furthermore, the combination of each variant of $\mathrm{C} 3$ preview with the next character $(\mathrm{C} 4)$ was not a word. The combination of the target and C3' in the ambiguous condition was not a 3-character word.

The sentences contained 26-27 characters. Target words were embedded at the 13th to 18th character positions. The punctuation marks (if any) were at least two characters to the left of the two-character pretarget words or 3 characters to the right of the target words. There was always a minimum of 6 characters succeeding the target. There were 24 trials in each of 6 conditions. Three lists were created, in

\footnotetext{
${ }^{2} \mathrm{C} 3$ in the identical condition was a single-character word, which is usually highly frequent and visually simple (in terms of number of strokes). Thus, $\mathrm{C} 3$ in the identical condition and those in the non-identical conditions were different in number of strokes and character frequency ( $p$ s $<.001)$. However, the critical comparison in the present study was between the two non-identical conditions, in which C3 was carefully matched $(p s>.99)$.

3 As is shown in Table 2, numbers of strokes of C1 and C2 did not differ between the low- and highprobability conditions $(p s>.58)$. Character frequencies of $\mathrm{C} 1$ did not differ between conditions $(p>.96)$. However, $\mathrm{C} 2 \mathrm{~s}$ in the low-probability condition $(M=198.7$ per 1 million, $\mathrm{SD}=239.8$, range 16.0-1222.1) were less frequent than those in the high-probability condition $(M=343.6$ per 1 million, $\mathrm{SD}=366.7$, range 24.2-1589.0), $p<.01$. Although they differed in frequencies of occurrence in the corpus of written characters, they were all subjectively familiar characters (5.3 and 5.3, respectively for the low- and high-probability conditions, $p>.49$ ). The subjective familiarity is based on an unpublished corpus of 5640 Chinese characters. The data were collected from 160 college students by using a 7-point scale for familiarity rating (Lee, Tsai, Chan, Hsu, Hung, \& Tzeng, 2007, p. 148). The subjective familiarity rating values of all characters in the critical $\mathrm{C} 123$ area were higher than 3.5 . In addition, when the item analysis was restricted to targets with $\mathrm{C} 2$ frequencies lower than 100 per 1 million (33 and 22 items in the low- and high-probability conditions, respectively), similar patterns of results were obtained on FFD, GD, and refixation rate in which the ROI was fixated longer and more likely refixated in the lowthan high-probability condition (C123: FFD, $8 \mathrm{~ms}, p>.32$, GD, $30 \mathrm{~ms}, p>.17$, refixation rate, $4.1 \%$, $p>.28$; C12: FFD, $8 \mathrm{~ms}, p>.34$, GD, $27 \mathrm{~ms}, p>.14$, refixation rate, $4.9 \%, p>.17)$. Nevertheless, we still have to be cautious about the interpretation of the effect of positional probability.
} 


\begin{abstract}
Sample sentence
為符合就業服務法, 公司徵人應該取消對求職者的年齒限制。

[Translation] To comply with the Employment Services Act, companies should

lift (cancel) the age restrictions of job applicants when hiring new employees.
\end{abstract}

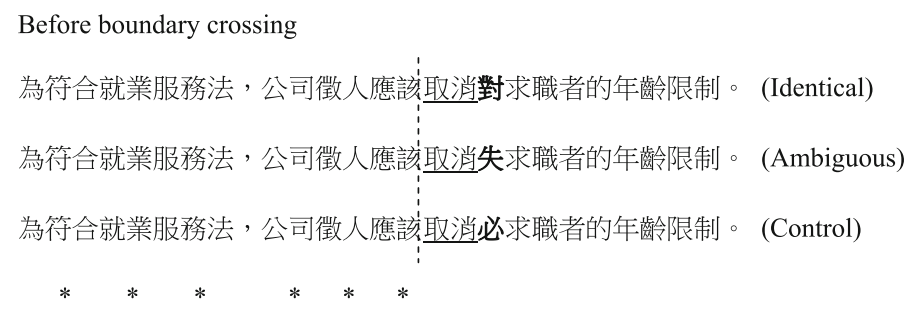

After boundary crossing

為符合就業服務法, 公司徵人應該取消對求職者的年齡限制。

Fig. 1 Illustration of the procedure with a sample sentence. The target word (C12) is underlined; $\mathrm{C} 3$ and the preview stimuli are presented in bold face for illustration

which each experimental sentence was assigned to only one of the three preview conditions for each participant. In other words, participants read each sentence only once. A sample sentence with the target and the preview stimuli is shown in Fig. 1.

\title{
Apparatus
}

The eye movements were recorded by the EYELINK II eye-tracking system, manufactured by SR Research, with a sampling rate of $500 \mathrm{~Hz}$. The sentences were displayed on a ViewSonic PT795 monitor. In the experiment program, a set of VGA routines from a PCTSCOPE library (Tsai, 2001) was used to increase the vertical refresh rate of the display to $167 \mathrm{~Hz}$ and preload all images into VGA memory before each trial. Eye-movement contingent display changes were accomplished by combining the PCTSCOPE library for fast display change and the EYELINK software for detecting eye positions on line. The overall time it took to acquire the current eye position and implement a display change was less than $12 \mathrm{~ms}$. During this process, the movement of the eye across the boundary was detected within $6 \mathrm{~ms}$. Then, the experiment control program initiated the display change by switching images in $40.5 \mu \mathrm{s}$. The display change was completed within one refresh cycle (range $0-6 \mathrm{~ms}$ ). 
The sentences were presented in black on a light gray background on the display monitor. The screen resolution of the display monitor was $800 \times 600$ pixels. The size of the character was $24 \times 24$ pixels and the size of the space between characters was $4 \times 24$ pixels. The viewing distance was $60 \mathrm{~cm}$, at which each character subtended $1.05^{\circ}$.

\section{Procedure}

Participants were instructed to read 180 sentences (36 of which were fillers) for comprehension at their normal pace. They were told that there would be a yes/no comprehension question after some of the sentences. After setting up the eye tracking system, a horizontal three-point calibration was conducted, followed by a validation routine that verified accuracy. Then, 12 practice trials were presented to ensure that participants understood the task. During the experiment, each trial started with a fixation point presented at the location of the first character of the sentence (the location was fixed regardless of sentence length). Participants read each sentence at their own pace and pressed a button to indicate that they finished reading and understood the sentence. During 30\% of the trials, a comprehension question was presented on the screen following the disappearance of the sentence. Participants were asked to decide whether the sentence in the comprehension test paraphrased the one they just read by pressing buttons. Participants were allowed to take a break after 60 trials. Calibration was conducted every 15 trials, after breaks, and when there was a drift from the fixation point presented prior to the experimental sentence. The experiment lasted about one hour.

The procedure of each trial is illustrated in Fig. 1. An invisible boundary was set at the first pixel of the space preceding the target word. At first, according to the condition, the sentence with the corresponding preview stimulus $(\mathrm{C} 3$, the character to the right of the target word) was displayed. After participants moved their eyes across the boundary, the display change was implemented so that the sentence with the original C3 was presented. In the filler sentences, the boundaries were set at 13th to 18 th character positions as if there were target words. The boundaries were not necessarily set prior to the beginning of a word (the length of which was not restricted). The pseudo-C3 was not necessary a single-character word; it replaced itself after boundary crossing so that there was no visible changes. Participants were instructed to ignore any disturbance that might occur while they were reading the experimental sentences. In the interview following the experiment, some participants reported having seen something flash or change $(M=2.6 \%$ of time, $\mathrm{SD}=1.3 \%, \max =5.6 \%$ ), but they did not know what had been presented before display change. The trials in which display changes were triggered improperly (see the "Results" for details) were excluded from analysis.

\section{Results}

The region of interest (ROI) in the present study is the critical area C123. Similar patterns of results were obtained when the target $\mathrm{C} 12$ was chosen as the ROI. 


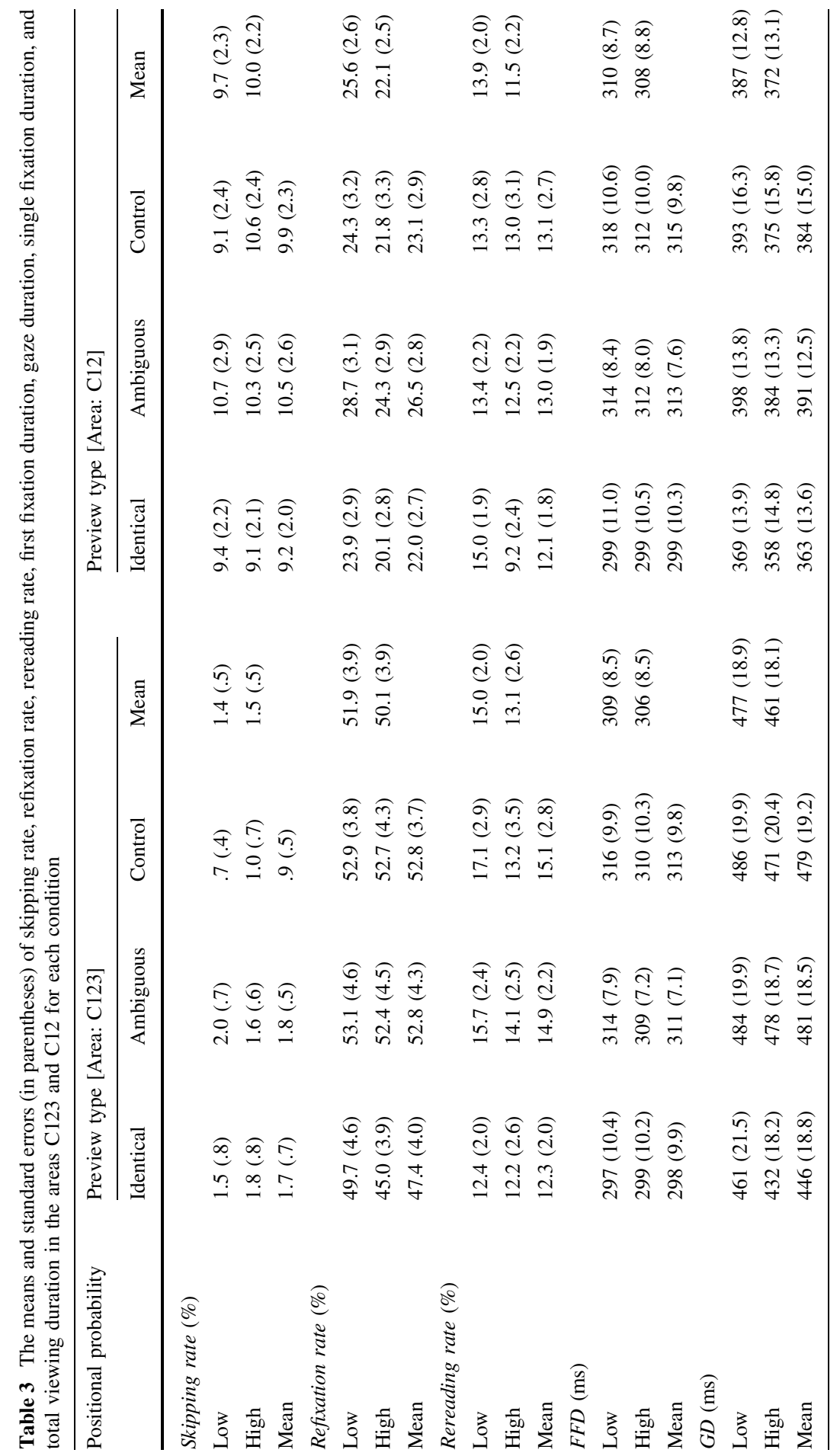




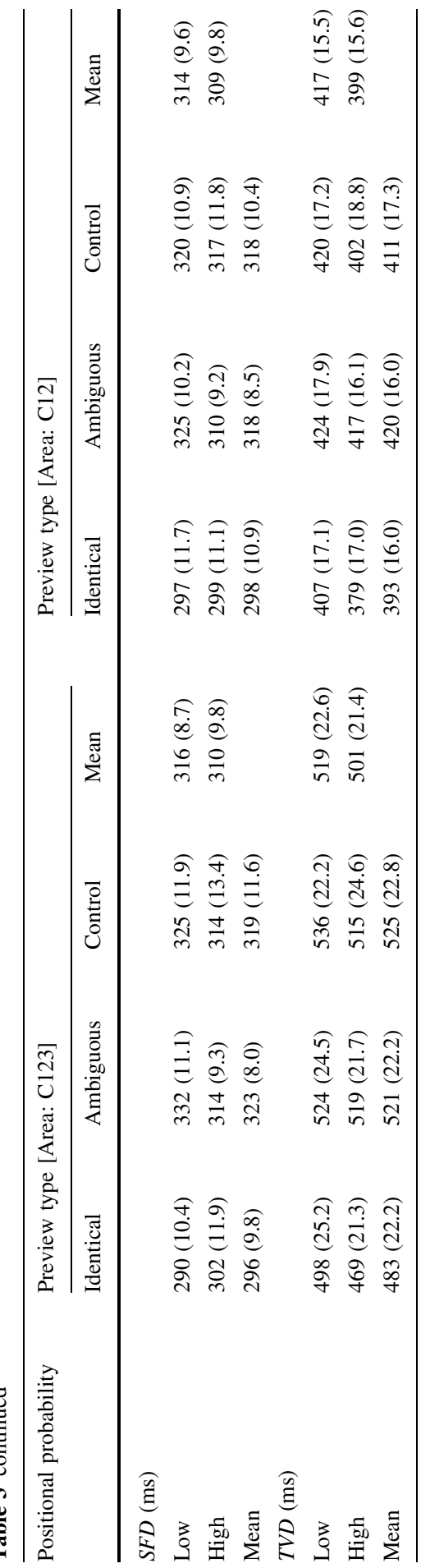


Table 4 The means and standard errors (in parentheses) of the probabilities of fixating C12 and C3 after the initial fixation in the area $\mathrm{C} 123$ and the probability of fixating $\mathrm{C} 3$ after leaving the target $\mathrm{C} 12$ for each condition

\begin{tabular}{|c|c|c|c|c|c|c|c|c|}
\hline \multirow{2}{*}{$\begin{array}{l}\text { Positional } \\
\text { probability }\end{array}$} & \multicolumn{4}{|c|}{ Preview type [After initial fixation on C123] } & \multicolumn{4}{|c|}{ Preview type [After leaving C12] } \\
\hline & Identical & Ambiguous & Control & Mean & Identical & Ambiguous & Control & Mean \\
\hline \multicolumn{9}{|c|}{ Fixating C12 } \\
\hline Low & $24.5(2.8)$ & $28.6(3.1)$ & $24.1(3.2)$ & $25.7(2.6)$ & & & & \\
\hline High & $20.3(2.7)$ & $23.7(3.0)$ & $22.0(3.1)$ & $22.0(2.5)$ & & & & \\
\hline Mean & $22.4(2.6)$ & $26.2(2.9)$ & $23.1(2.8)$ & & & & & \\
\hline \multicolumn{9}{|l|}{ Fixating $C 3$} \\
\hline Low & $25.3(2.6)$ & $24.5(2.6)$ & $28.8(2.4)$ & $26.2(2.0)$ & $33.3(3.5)$ & $33.4(3.3)$ & $36.6(3.2)$ & $34.4(2.8)$ \\
\hline High & $24.7(2.3)$ & $28.7(3.1)$ & $30.7(3.3)$ & $28.1(2.4)$ & $30.6(2.8)$ & $35.6(3.6)$ & $38.3(3.6)$ & $34.8(2.9)$ \\
\hline Mean & $25.0(2.0)$ & $26.6(2.5)$ & $29.8(2.4)$ & & $31.9(2.7)$ & $34.5(3.2)$ & $37.4(3.1)$ & \\
\hline
\end{tabular}

Results of both regions are shown in Table 3, but the statistics are reported only for the area $\mathrm{C} 123$ to avoid redundancy. There was an interesting difference in the pattern of refixation rate between the area $\mathrm{C} 123$ and the target $\mathrm{C} 12$. To further elucidate this difference, a detailed analysis about the probabilities of fixating $\mathrm{C} 12$ and C3 (Table 4) was conducted to examine the locus of the effects of positional probability and ambiguous preview. ${ }^{4}$

Four first-pass measures (i.e., gaze duration, first fixation duration, single fixation duration, and refixation rate) in the ROIs were calculated. Gaze duration (GD) was used as the primary measure. It is the sum of the durations of all first-pass fixations in the ROI before leaving it. First fixation duration (FFD) is the duration of the first first-pass fixation in the ROI regardless of how many fixations were made, and single fixation duration (SFD) is the duration in the ROIs which received only one first-pass fixation. Refixation rate in each condition is the proportion of trials in which the ROI received more than one first-pass fixation. In addition, the proportion of trials in which the ROI was not fixated during first-pass reading (skipping rate), the proportion of trials in which the ROI was reread after the eyes moved to the right (rereading rate), and the sum of all fixation durations, including second-pass fixations, in the ROI (total viewing duration, TVD) were calculated as supplementary measures. A 2 (C2 positional probability: high and low) $\times 3$ (C3 preview type: identical, ambiguous, and control) ANOVA was performed for each measure separately.

Data analysis was restricted to trials in which the two-character word (pretarget) prior to the target $\mathrm{C} 12$ was fixated during first-pass reading and the eyes moved rightwards after leaving the pretarget. Two percent of trials were excluded because the participants blinked when they fixated the pretarget or the area C123. Additional

\footnotetext{
${ }^{4}$ It would be informative to analyze data separately for the target $\mathrm{C} 12$ and the preview $\mathrm{C} 3$ to examine the time course of the effects of positional probability and preview type (we thank one of the reviewers for this suggestion). However, $\mathrm{C} 3$ was a single-character word with high skipping rate. The data points were too few to have reliable statistics. Thus, C123 was chosen as the ROI with sufficient data points to be analyzed.
} 
$11 \%$ of trials were excluded because the pretarget was skipped during the first-pass reading; five percent of trials were excluded because the eyes moved leftwards after leaving the pretarget; and a few trials $(.3 \%)$ were excluded because gaze durations on the pretarget were shorter than $100 \mathrm{~ms}$. Then, six percent of trials were excluded because the display changes were triggered later than $10 \mathrm{~ms}$ after fixation onset. Skipping rate, rereading rate and TVD were calculated with the remaining trials (76\% in total). The four first-pass measures were calculated with trials in which each ROI was fixated immediately after first-pass fixations on the pretarget. Then, viewing durations shorter than $80 \mathrm{~ms}$ or longer than 1,200 ms were excluded from analysis. This cutoff was applied to each duration measures (GD, FFD, SFD, and TVD) separately. About $75 \%$ of trials were retained for FFD, GD, and TVD in the area $\mathrm{C} 123$. For SFD, $36 \%$ of trials were available. Note that due to the relatively large number of trials ( 24 per cell), removal of data did not result in any empty cells within the 2 by 3 design. However, two participants were replaced because there were less than 9 valid trials in a few conditions in their data sets. The means and standard errors of skipping rate, refixation rate, rereading rate, FFD, GD, SFD, and TVD in each condition are shown in Table 3.

\section{Skipping rate}

The area $\mathrm{C} 123$ were skipped about $1.5 \%$ of time. There was neither a probability effect $\left[-.1 \% ; F_{1}(1,26)<1, M S_{\mathrm{e}}=6 ; F_{2}(1,142)<1, M S_{\mathrm{e}}=36\right]$ nor a preview effect $\left[F_{1}(2,52)=1.51, M S_{\mathrm{e}}=9, p>.23 ; F_{2}(2,284)=2.01, M S_{\mathrm{e}}=24, p>.13\right]$ on fixation probability. The interaction was also negligible $(F \mathrm{~s}<1)$.

\section{Viewing duration measures}

A substantial effect of positional probability was observed on gaze durations. GDs in the area $\mathrm{C} 123$ were longer in the low-probability condition than those in the high-probability condition $\left[16 \mathrm{~ms} ; F_{1}(1,26)=4.52, M S_{\mathrm{e}}=2365, p<.05\right.$; $\left.F_{2}(1,142)=1.92, M S_{\mathrm{e}}=17002, p>.16\right]$. A similar pattern was observed for TVDs $\left[18 \mathrm{~ms} ; F_{1}(1,26)=4.26, M S_{\mathrm{e}}=3102, p<.05 ; F_{2}(1,142)=2.23\right.$, $\left.M S_{\mathrm{e}}=24462, p>.13\right]$. However, the probability effects on FFD [3 ms; $\left.F_{1}(1,26)<1, M S_{\mathrm{e}}=424 ; F_{2}(1,142)<1, M S_{\mathrm{e}}=2611\right]$ and SFD [6 ms; $\left.F_{1}(1,26)<1, M S_{\mathrm{e}}=1572 ; F_{2}(1,135)<1, M S_{\mathrm{e}}=6419\right]$ were not significant.

Significant effects of preview type were observed in all duration measures, for FFD $\left[F_{1}(2,52)=4.55, M S_{\mathrm{e}}=871, p<.05 ; F_{2}(2,284)=6.14, M S_{\mathrm{e}}=1586\right.$, $p<.01]$, GD $\left[F_{1}(2,52)=9.22, M S_{\mathrm{e}}=2194, p<.001 ; F_{2}(2,284)=7.70\right.$, $\left.M S_{\mathrm{e}}=6818, p<.01\right], \mathrm{SFD}\left[F_{1}(2,52)=6.50, M S_{\mathrm{e}}=1786, p<.01 ; F_{2}(2,270)=\right.$ $\left.7.26, M S_{\mathrm{e}}=4525, p<.01\right]$, and TVD $\left[F_{1}(2,52)=9.51, M S_{\mathrm{e}}=3029, p<.001\right.$; $\left.F_{2}(2,284)=10.73, M S_{\mathrm{e}}=6192, p<.001\right]$. Post-hoc pairwise comparisons revealed that viewing durations were significantly shorter in the identical condition than those in the control condition [FFD $16 \mathrm{~ms}, p<.05$; GD $32 \mathrm{~ms}, p<.05$; SFD $23 \mathrm{~ms}, p<.05$; and TVD $42 \mathrm{~ms}, p<.01]$. Viewing durations were also significantly shorter in the identical condition than those in the ambiguous condition 
[FFD $14 \mathrm{~ms}, p=.050$; GD $35 \mathrm{~ms}, p<.001$; SFD $27 \mathrm{~ms}, p<.01$; and TVD $38 \mathrm{~ms}$, $p<.001]$. However, the difference between the ambiguous and the control conditions were almost negligible [FFD: $-2 \mathrm{~ms}$, GD $2 \mathrm{~ms}$, SFD $4 \mathrm{~ms}$, and TVD: 4 ms. All $p$ s $>$.99].

The interaction between positional probability and preview type on TVD was marginally significant by items $\left[F_{1}(2,52)=1.10, M S_{\mathrm{e}}=1826, p>.34 ; F_{2}(2\right.$, $\left.284)=2.73, M S_{\mathrm{e}}=6192, p=.067\right]$. The interaction on other duration measures were not significant (all $F \mathrm{~s}<1.8$ and $p \mathrm{~s}>.17$ ).

\section{Refixation rate}

The area C123 was slightly more frequently refixated in the low-probability condition than that in the high-probability condition, but the probability effect was not significant $\left[1.9 \% ; F_{1}(1,26)=1.37, M S_{\mathrm{e}}=104, p>.25 ; F_{2}(1,142)<1\right.$, $\left.M S_{\mathrm{e}}=579\right]$. In contrast, a significant effect of preview type was observed $\left[F_{1}(2\right.$, $\left.52)=4.40, M S_{\mathrm{e}}=121, p<.05 ; F_{2}(2,284)=3.44, M S_{\mathrm{e}}=374, p<.05\right]$. The area C123 was more likely refixated in the ambiguous $(52.8 \%)$ and the control $(52.8 \%)$ conditions than that in the identical condition (47.4\%), both $p \mathrm{~s}<.05$. The $.03 \%$ difference between the ambiguous and control conditions was negligible $(p>.99)$. The interaction was not significant $(F \mathrm{~s}<1)$.

To the contrary, a significant probability effect was observed on the target $\mathrm{C} 12$ $\left[3.6 \% ; F_{1}(1,26)=8.42, M S_{\mathrm{e}}=61, p<.01 ; F_{2}(1,142)=4.74, M S_{\mathrm{e}}=491\right.$, $p<.05]$. However, there was neither an effect of preview type $\left[F_{1}(2,52)=2.05\right.$, $\left.M S_{\mathrm{e}}=146, p>.13 ; F_{2}(2,284)=2.99, M S_{\mathrm{e}}=302, p=.052\right]$ nor an interaction $(F \mathrm{~s}<1)$.

\section{Fixation probability on $\mathrm{C} 12$ and C3}

The mean initial landing position in the area $\mathrm{C} 123$ was slightly to the right of $\mathrm{C} 1$ (1.04 character position). The probabilities of initially landing on $\mathrm{C} 1, \mathrm{C} 2$, and $\mathrm{C} 3$ were $51.5,39.8$, and $8.7 \%$, respectively. There were no effects of $\mathrm{C} 2$ probability and $\mathrm{C} 3$ preview on the initial landing probabilities $(F \mathrm{~s}<2, p \mathrm{~s}>.23)$.

The probabilities of fixating $\mathrm{C} 12, \mathrm{C} 3$, or leaving $\mathrm{C} 123$ after the initial fixation in C123 were calculated. As is shown in Table 4, the probability of fixating C12 after initially landing on $\mathrm{C} 123$ was higher in the low-probability (25.7\%) than that in the high-probability $(22.0 \%)$ condition $\left[F_{1}(1,26)=10.24, M S_{\mathrm{e}}=55, p<.01\right.$; $\left.F_{2}(1,142)=4.25, M S_{\mathrm{e}}=500, p<.05\right]$. The effect of preview type was negligible $(F \mathrm{~s}<2.2, p \mathrm{~s}>.11)$ and there was no interaction $(F \mathrm{~s}<1)$. On the other hand, there was no effect of positional probability on the probability of fixating $\mathrm{C} 3(F \mathrm{~s}<1.6$, $p \mathrm{~s}>.22)$. But a significant effect of preview type was observed $\left[F_{1}(2,52)=3.34\right.$, $\left.M S_{\mathrm{e}}=95, p<.05 ; F_{2}(2,284)=2.82, M S_{\mathrm{e}}=323, p=.062\right]$. The difference between the identical and control condition was marginally significant ( $p s>.06)$. There was no interaction $(F \mathrm{~s}<1.1, p \mathrm{~s}>.35)$.

Similar patterns were observed when the target $\mathrm{C} 12$ was chosen as the ROI. As mentioned above, the probability of refixating $\mathrm{C} 12$ was only influenced by 
positional probability of $\mathrm{C} 2$. In contrast, the probability of fixating $\mathrm{C} 3$ after leaving $\mathrm{C} 12$ was influenced by preview type $\left[F_{1}(2,52)=3.65, M S_{\mathrm{e}}=112, p<.05 ; F_{2}(2\right.$, $\left.284)=3.31, M S_{\mathrm{e}}=409, p<.05\right]$. A significant difference between the identical and the control conditions $(p s<.05)$ was observed. There was neither an effect of positional probability nor an interaction (all $F \mathrm{~s}<1.38, p \mathrm{~s}>.25$ ).

\section{Rereading rate}

The area C123 was slightly more likely reread in the low-probability than highprobability conditions but the probability effect was not significant $\left[1.9 \% ; F_{1}(1\right.$, $\left.26)=1.80, M S_{\mathrm{e}}=82, p>.19 ; F_{2}(1,142)=2.02, M S_{\mathrm{e}}=247, p>.15\right]$. A marginally significant effect of preview type was observed by participants $\left[F_{1}(2\right.$, $\left.52)=2.75, M S_{\mathrm{e}}=49, p=.073 ; F_{2}(2,284)=2.22, M S_{\mathrm{e}}=156, p>.11\right]$ The area C123 was slightly more likely reread in the ambiguous $(14.9 \%)$ and control $(15.1 \%)$ conditions than that in the identical condition (12.3\%), $p<.05$ and $p>.28$, respectively. The difference between the ambiguous and control conditions was negligible, $p>.99$. The interaction between positional probability and preview type was negligible $\left(F_{\mathrm{s}}<1\right)$.

\section{Discussion}

Significant effects of positional probability and preview type on gaze durations in the critical area $\mathrm{C} 123$ were observed; but there was no interaction between these factors. Gaze durations were substantially longer in the low-probability condition than those in the high-probability condition. The result suggests that it is difficult to process target words if their ending characters are more frequently used as word beginning than as word ending. Concerning the manipulation of preview type, viewing durations were lengthened by non-identical previews, in which the ambiguous and control stimuli were equally effective. Viewing durations in the ambiguous condition were not significantly longer than those in the control condition, suggesting that overlapping ambiguity between parafoveal words may not have been detected during preview. In addition, the positional probability of $\mathrm{C} 2$ did not modulate the preview effect of $\mathrm{C} 3$ and the activation of the overlapping word C23.

The observation that effects of C2 probability and C3 preview were fairly independent is further supported by the patterns of fixation probability within the area $\mathrm{C} 123$. Concerning the second fixation within this area, the probability of fixating $\mathrm{C} 12$ was mainly influenced by $\mathrm{C} 2$ probability, while the probability of fixating $\mathrm{C} 3$ was solely influenced by $\mathrm{C} 3$ preview. The higher refixation rate on the target $\mathrm{C} 12$ in the low-probability condition than that in the high-probability condition may largely account for the observation that gaze durations on the target in the former condition were longer than those in the latter condition [15 ms; $\left.F_{1}(1,26)=9.31, M S_{\mathrm{e}}=937, p<.01 ; F_{2}(1,142)=2.22, M S_{\mathrm{e}}=12134, p>.13\right]$ because there was no difference in FFDs between conditions $\left(3 \mathrm{~ms} ; F_{\mathrm{s}}<1\right)$. 
The findings that overlapping ambiguity between parafoveal words did not have an effect and that the positional probability of $\mathrm{C} 2$ did not interact with the preview type of C3 imply that the ambiguity in word boundaries and positional probability were mainly processed during foveal processing. This implication is further manifested by the comparison of the results of Inhoff and Wu, (2005) to that of the present study. The ambiguous character strings in their study were presented throughout sentence reading while those in the present study were presented before first-pass fixations on the strings. The presence of the ambiguity effect in the study of Inhoff and $\mathrm{Wu}$ and the absence of this effect in the present study implies that ambiguity in word segmentation is processed foveally rather than parafoveally. The perceptual span in Chinese reading is about 1 character to the left and 3 characters to the right of the fixated character (Inhoff \& Liu, 1998) which may contain a twocharacter foveal word (e.g., the pretarget in the present study), a two-character parafoveal word (the target) and one more character (C3). Although all characters in the span can be processed, segmenting all characters in the span into words demands effort because there is some uncertainty in character-to-word assignment (i.e., which character belongs to which word). Both eccentricity and uncertainty in word segmentation contribute to the observation that, during preview, overlapping ambiguity between parafoveal words did not have an effect and that only one parafoveal word $n+1$ can be processed (Yang et al., 2009).

Concerning the time course of positional probability, lack of interaction with C3 preview suggests that it plays a role mainly during foveal processing. For clarification, the rationale of the present experimental design is recapitulated and elaborated in details. If $\mathrm{C} 2$ is more likely to be word beginning, it should be easier to activate the overlapping word that begins with $\mathrm{C} 2$ than if $\mathrm{C} 2$ is more likely to be word ending. If the positional probability of $\mathrm{C} 2$ is available during preview, in the low-probability condition, it can be expected to augment the activation of the overlapping word C23 and then induce the parafoveal ambiguity effect. Alternatively, if the overlapping word could be activated during preview, then the competition between $\mathrm{C} 12$ and $\mathrm{C} 23$ should differ because of the positional probability of $\mathrm{C} 2$. Following either viewpoint, the ambiguity effect on viewing durations should be larger in the low-probability condition than that in the highprobability condition. In addition, the probabilities of fixating C12 and C3 should be influenced by both the probability of $\mathrm{C} 2$ and the preview of $\mathrm{C} 3$. However, no such interaction was observed, indicating that the positional probabilities of parafoveal characters may not be available during preview or may have their effects mainly during foveal processing. Positional probability manifested its effect by lengthening target gaze durations and increasing refixation rate in the low-probability condition. The target is difficult to process if any of its constituent characters is located at an infrequent within-word position. The effect of positional probability was not observed on FFD but was observed on refixation rate and GD, which implies that positional probability may not have its effect early enough to influence the duration of the initial fixation (c.f., Bertram et al., 2004).

The task of word recognition during reading Chinese sentences is complicated by the fact that there are no visual cues for word boundaries. It is difficult to recognize a word in a series of characters also because most constituent characters can appear 
at multiple within-word positions. There is uncertainty about whether a character should be combined with character(s) to its right or left to form a word in the context. Grouping characters into words may be easy if the connection between constituent characters is strong. For example, there are binding words in which the constituents always co-occur with each other to form this single word. These words should be easy to identify in the continuous text. Besides the special case of binding words (which have no orthographic neighbor), most characters can be combined with other characters to form different words. The size of orthographic neighborhood (words sharing constituent letters; see Andrews, 1997, for a review) and that of morphological family (words sharing a base word; Bertram, Baayen, \& Schreuder, 2000) have been shown to influence word recognition. How these factors affect the connection strength between constituent characters is worth further investigation (see Tsai, et al., 2006, for an example). Besides the connection between the constituent characters, whether or not a constituent character signals a word boundary to its left or right is also helpful for recognizing words embedded in the sentence. The present study aimed to investigate whether positional probability has an effect on word recognition. If a character is proportionally more likely to be word ending than word beginning, it may signal a word boundary to its right. In fact, the effect of positional probability on target viewing durations was observed in the present study. However, as is discussed above, this effect may mainly occur during foveal processing. In addition, subjective familiarity and objective frequency of words and characters should be taken into consideration. The critical character $\mathrm{C} 2 \mathrm{~s}$ were equally familiar between the low- and high-probability conditions according to a corpus of subjective familiarity rating; ${ }^{3}$ however, the objective frequencies of $\mathrm{C} 2 \mathrm{~s}$ in the low-probability condition were lower than those in the high-probability condition. When the item analysis was restricted to low frequency characters (lower than 100 per 1 million), the patterns of results were similar to those of the main analysis. Nevertheless, we still have to be cautious about the interpretation of the effect of positional probability. Future studies that simultaneously compare the effects of positional probability and frequency are necessary for clarification.

What can be processed from the perceptual span in Chinese reading? Yen et al. (2008) have shown that a word can be activated from the parafovea (also see Yang et al., 2009). However, only character-level information can be obtained from the remaining area (e.g., C3) farther in the parafovea. Whether or not C3 can be combined with $\mathrm{C} 2$ to form another word did not influence parafoveal processing. When a word (which may be activated during preview) was fixated, character-toword assignment (i.e., which character belongs to which word) was verified, during which the probability of within-word positions of each character was taken into consideration. If a character that is frequently used as word ending was indeed presented at the ending position, the consistency in within-word positions could facilitate word recognition in continuous text. Thus, the target word was less likely to be refixated and gaze duration on the target was shortened. If overlapping ambiguity was present during fixation, viewing durations on the ambiguous string would be lengthened (Inhoff $\& \mathrm{Wu}, 2005$ ). In this situation, context is essential to resolve the ambiguity. 
Computational models of eye movement control in reading that generally assume word-based processing should be modified to incorporate the characteristic that Chinese words are not visually segmented. The findings of the present study indicate that the probability of within-word positions of each character has an influence on character-to-word assignment, which is mainly verified during foveal processing. Further research on the time course of using statistical cues for the delineation of word boundaries appears necessary. In addition, the relative contribution of various factors, such as character/word frequencies, positional probability, and neighborhood size, should be systematically compared in future studies. Current computational models can then incorporate the probability of within-word positions together with other linguistic factors in their word processing systems. As an example, within the interactive activation letter processing framework of the Glenmore Model (Reilly \& Radach, 2006), statistical properties of character locations can be used to substitute visual word boundaries as soon as feedback from character level processing becomes available. In addition, it can be tested whether adding the properties of positional probability improves model performance when applying word-based processing mechanism to Chinese reading in the E-Z Reader model (Rayner et al., 2007).

The findings of the present study can provide some suggestions about the highly debated issue of serial or distributed processing. This issue is more complicated in reading Chinese than alphabetic scripts because both characters and words have meanings and are functional units in Chinese script. Thus, how processing of characters and words interact with each other and how words are segmented from a series of characters are critical issues. Because words are not visually segmented and most characters can be used as word beginning and word ending, processing of characters in the perceptual span will likely have to overlap in time for word segmentation. Li, Rayner and Cave (2009) proposed that characters within the span are processed in parallel, with processing efficiency decreasing according to eccentricity. Furthermore, Yen et al. (2009) observed a beginning-of-fixation visibility effect of parafoveal characters on foveal words. If the parafoveal characters were masked during the initial $140 \mathrm{~ms}$ after fixation onset, the foveal words were fixated longer than those in the condition in which the parafoveal characters were visible. This finding suggests that early parafoveal processing, at least at the character level, is helpful for foveal word processing. It also suggests that characters within the span are processed in parallel.

This issue of serial versus distributed processing of words is still puzzling. Both eccentricity and uncertainty in word boundaries can contribute to the observation that only one parafoveal word can be processed (the present study and Yang et al. 2009). However, the overlapping ambiguity effect observed in the study of Inhoff and $\mathrm{Wu}$ (2005) suggests that processing of the foveal word and parafoveal word may overlap in time. The time course of word recognition/segmentation during foveal processing (early or late) deserves further investigation. In addition to clarifying the mechanism of recognizing unsegmented Chinese words, it is intriguing to ask what kind of information is involved in saccade target selection during Chinese reading. For example, positional probability has been shown to influence saccade targeting in Thai, but it did not influence the initial landing 
position in the present study. Further research is necessary to clarify this discrepancy. However, the fact that number of Chinese characters is much more than number of Thai alphabets should be taken into consideration for this issue.

To conclude, although there is no physical cue for word boundaries in Chinese, readers can use statistical cues for word segmentation and recognition. Inconsistency in the frequent within-word position (word beginning) and the present position (word ending) in the target word increased refixation rate and lengthened gaze durations on the targets. However, the probability of within-word positions of each character may be effective mainly during foveal processing; thus, the presence of an overlapping ambiguity between parafoveal words did not interfere with subsequent reading performance. Taken together, the results of the present study suggest that statistical properties of characters are important for word recognition and eye movement control during Chinese reading.

Acknowledgments This study was supported by the grants from Taiwan's National Science Council (NSC96-2413-H-004-018-MY3, NSC 99-2420-H-004-002-, NSC98-2811-H-004-022, NSC 99-2811-H004-015, and NSC 100-2410-H-003-001-). The final version of this manuscript was completed when the first author moved to the Graduate Institute of Science Education, National Taiwan Normal University. We thank two anonymous reviewers for helpful comments on earlier versions of the manuscript.

\section{References}

Academia Sinica Taiwan. (1998). Academia Sinica balanced corpus (Version 3) [CD-ROM]. Taipei, Taiwan: Academia Sinica, Chinese Knowledge and Information Processing Group.

Andrews, S. (1997). The effect of orthographic similarity on lexical retrieval: Resolving neighborhood conflicts. Psychonomic Bulletin \& Review, 4, 439-461.

Baayen, R. H. (2008). Analyzing linguistic data: A practical introduction to statistics using R. Cambridge, UK: Cambridge University Press.

Bertram, R., Baayen, R. H., \& Schreuder, R. (2000). Effects of family size for complex words. Journal of Memory and Language, 42, 390-405.

Bertram, R., Pollatsek, A., \& Hyönä, J. (2004). Morphological parsing and the use of segmentation cues in reading Finnish compounds. Journal of Memory and Language, 51, 325-345.

Engbert, R., Nuthmann, A., Richter, E. M., \& Kliegl, R. (2005). SWIFT: A dynamical model of saccade generation during reading. Psychological Review, 112, 777-813.

Findlay, J. M., \& Gilchrist, I. D. (2003). Active Vision: the Psychology of Looking and Seeing. Oxford: Oxford University Press.

Inhoff, A. W. (1984). Two stages of word processing during eye fixations in the reading of prose. Journal of Verbal Learning and Verbal Behavior, 23, 612-624.

Inhoff, A. W., \& Liu, W. (1998). The perceptual span and oculomotor activity during the reading of Chinese sentences. Journal of Experimental Psychology: Human Perception and Performance, 24, 20-34.

Inhoff, A. W., \& Wu, C. (2005). Eye movements and the identification of spatially ambiguous words during Chinese sentence reading. Memory \& Cognition, 33, 1345-1356.

Lee, C.-Y., Tsai, J.-L., Chan, W.-H., Hsu, C.-H., Hung, D. L., \& Tzeng, O. J.-L. (2007). Temporal dynamics of the consistency effect in reading Chinese: an event-related potentials study. NeuroReport, 18, 147-151.

Li, X., Rayner, K., \& Cave, K. R. (2009). On the segmentation of Chinese words during reading. Cognitive Psychology, 58, 525-552.

Liu, W., Inhoff, A. W., Ye, Y., \& Wu, C. (2002). Use of parafoveally visible characters during the reading of Chinese sentences. Journal of Experimental Psychology: Human Perception and Performance, 28, 1213-1227. 
McConkie, G. W., Kerr, P. W., Reddix, M. D., \& Zola, D. (1988). Eye movement control during reading: I. The location of initial eye fixations on words. Vision Research, 28, 1107-1118.

McConkie, G. W., \& Rayner, K. (1975). The span of the effective stimulus during a fixation in reading. Perception \& Psychophysics, 17, 578-586.

McDonald, S. A., \& Shillcock, R. C. (2003). Low-level predictive inference in reading: The influence of transitional probabilities on eye movements. Vision Research, 43, 1735-1751.

Pollatsek, A., Reichle, E. D., \& Rayner, K. (2006). Tests of the E-Z Reader model: Exploring the interface between cognition and eye-movement control. Cognitive Psychology, 52, 1-56.

Radach, R., Reilly, R., \& Inhoff, A. W. (2007). Models of oculomotor control in reading: Towards a theoretical foundation of current debates. In R. P. G. van Gompel, M. H. Fischer, W. S. Murray, \& R. L. Hill (Eds.), Eye movements: A window on mind and brain (pp. 237-269). Oxford: Elsevier.

Rayner, K. (1975). The perceptual span and peripheral cues in reading. Cognitive Psychology, 7, 65-81.

Rayner, K. (1979). Eye guidance in reading: Fixation locations within words. Perception, 8, 21-30.

Rayner, K. (1998). Eye movements in reading and information processing: 20 years of research. Psychological Bulletin, 124, 372-422.

Rayner, K., \& Duffy, S. A. (1986). Lexical complexity and fixation times in reading: Effects of word frequency, verb complexity, and lexical ambiguity. Memory \& Cognition, 14, 191-201.

Rayner, K., Li, X., Juhasz, B. J., \& Yan, G. (2005). The effect of word predictability on the eye movements of Chinese readers. Psychonomic Bulletin \& Review, 12, 1089-1093.

Rayner, K., Li, X., \& Pollatsek, A. (2007). Extending the E-Z Reader model of eye movement control to Chinese readers. Cognitive Science, 31, 1021-1033.

Rayner, K., \& Well, A. D. (1996). Effects of contextual constraint on eye movements in reading: A further examination. Psychonomic Bulletin \& Review, 3, 504-509.

Rayner, K., Well, A. D., \& Pollatsek, A. (1980). Asymmetry of the effective visual field in reading. Perception \& Psychophysics, 27, 537-544.

Rayner, K., Well, A. D., Pollatsek, A., \& Bertera, J. H. (1982). The availability of useful information to the right of fixation in reading. Perception \& Psychophysics, 31, 537-550.

Reilly, R. G., \& Radach, R. (2006). Some empirical tests of an interactive activation model of eye movement control in reading. Cognitive Systems Research, 7, 34-55.

Reilly, R. G., Radach, R., Luksaneeyanawin, S., \& Aranyanak, I. (2009, August). Factors involved in eye guidance in reading Thai. Paper presented at the 15th European Conference on Eye Movements, Southampton, UK.

Schilling, H. E. H., Rayner, K., \& Chumbley, J. I. (1998). Comparing naming, lexical decision, and eye fixation times: Word frequency effects and individual differences. Memory \& Cognition, 26, $1270-1281$.

Tsai, J.-L. (2001). A multichannel PC tachistoscope with high resolution and fast display change capability. Behavior Research Methods, Instruments, \& Computers, 33, 524-531.

Tsai, J.-L., Lee, C.-Y., Lin, Y.-C., Tzeng, O. J.-L., \& Hung, D. L. (2006). Neighborhood size effects of Chinese words in lexical decision and reading. Language and Linguistics, 7, 659-675.

Tsai, J.-L., Lee, C.-Y., Tzeng, O. J.-L., Hung, D. L., \& Yen, N.-S. (2004). Use of phonological codes for Chinese characters: Evidence from processing of parafoveal preview when reading sentences. Brain and Language, 91, 235-244.

Tsai, J.-L., \& McConkie, G. W. (2003). Where do Chinese readers send their eyes? In J. Hyönä, R. Radach, \& H. Deubel (Eds.), The mind's eye: Cognitive and applied aspects of eye movement research (pp. 159-176). Amsterdam, The Netherlands: Elsevier.

Underwood, N. R., \& McConkie, G. W. (1985). Perceptual span for letter distinctions during reading. Reading Research Quarterly, 20, 153-162.

Yan, M., Kliegl, R., Richter, E. M., Nuthmann, A., \& Shu, H. (2010). Flexible saccade-target selection in Chinese reading. Quarterly Journal of Experimental Psychology, 63, 705-725.

Yan, M., Richter, E. M., Shu, H., \& Kliegl, R. (2009). Readers of Chinese extract semantic information from parafoveal words. Psychonomic Bulletin \& Review, 16, 561-566.

Yan, G., Tian, H., Bai, X., \& Rayner, K. (2006). The effect of word and character frequency on the eye movements of Chinese readers. British Journal of Psychology, 97, 259-268.

Yang, H.-M., \& McConkie, G. W. (1999). Reading Chinese: Some basic eye-movement characteristics. In J. Wang, A. W. Inhoff, \& H.-C. Chen (Eds.), Reading Chinese script: A cognitive analysis (pp. 207-222). Mahwah, NJ: Lawrence Erlbaum Associates. 
Yang, J., Wang, S., Xu, Y., \& Rayner, K. (2009). Do Chinese readers obtain preview benefit from word $\mathrm{n}+2$ ? Evidence from eye movements. Journal of Experimental Psychology: Human Perception and Performance, 35, 1192-1204.

Yen, M.-H., Radach, R., Tzeng, O. J.-L., Hung, D. L., \& Tsai, J.-L. (2009). Early parafoveal processing in reading Chinese sentences. Acta Psychologica, 131, 24-33.

Yen, M.-H., Tsai, J.-L., Tzeng, O. J.-L., \& Hung, D. L. (2008). Eye movements and parafoveal word processing in reading Chinese. Memory and Cognition, 36, 1033-1045. 